\title{
The Regulation of YY1 in Tumorigenesis and its Targeting Potential in Cancer Therapy
}

\author{
Guangchao Sui
}

\author{
Department of Cancer Biology and Comprehensive Cancer Center, Wake Forest University \\ School of Medicine, Winston-Salem, North Carolina
}

\begin{abstract}
Yin Yang 1 (YY1) is a multifunctional protein and regulates various processes of development and differentiation. Increasing evidence indicates an essential role of YY1 in tumorigenesis. As a transcription factor, YY1 regulates the expression of numerous genes that are mostly involved in cancers. YY1 can either activate or repress the target genes, depending on the cofactors that it recruits. Importantly, most studies to date suggest a proliferative or oncogenic role of YY1 in cancer development. Meanwhile, overexpression of YY1 has been observed in different types of cancers and YY1 has been proposed as a potential prognostic marker of these cancers. A reasonable hypothesis is that upregulated YY1 leads to unbalanced expression of its target genes and in turn initiates or arguments tumorigenesis. Ample studies indicate that YY1 exerts broad regulation in various epigenetic events, especially histone acetylation and methylation. Since most cancers exhibit deregulated epigenetics, overexpressed YY1 may contribute to these aberrant epigenetic statuses in cancer cells. The epigenetic processes regulated by YY1 are reversible. Therefore, it is possible that targeting YY1 may adjust various deregulated epigenetic events in cancer cells, restore the normal epigenetic conditions and consequently block cancer development. This review summarizes cancer-related studies of YY1 and discusses the potential of YY1 as a target of cancer therapy.
\end{abstract}

Keywords: Cancer therapy; Epigenetic regulation; Tumorigenesis; YY1

Received 06/04/09; accepted 06/24/09

Correspondence: Guangchao Sui, Ph.D., Department of Cancer Biology, Wake Forest University School of Medicine, Hanes 4052, Medical Center Boulevard, Winston-Salem, NC 27157, USA. Tel. 336-713-0052, Fax. 336-716-0255. email: gsui@wfubmc.edu

\section{Introduction}

Aberrant expression and function of transcription factors play vital roles in oncogenic transformation of cells in different tissues. These factors interact with specific DNA elements and other protein cofactors to form transcriptional machinery that can either activate or repress the expression of essential genes involved in tumorigenesis. The altered or enhanced functions of these factors contribute, either directly or indirectly through other downstream pathways, to some or all of the cancer hallmarks, including insensitivity to antigrowth or apoptotic signals, self-sufficient growth signals, sustained angiogenesis, limitless replicative potential and invasive or metastatic capability (1).

Yin Yang 1 (YY1) is a ubiquitously expressed protein in all tissues and highly conserved in many different species. YY1-related studies keep accumulating and have branched to almost all existing research areas with over 700 papers available to date. Therefore, it is unrealistic to embrace all of these publications and even every research field in this review. For a comprehensive understanding of YY1 gene, protein and its regulatory models, please refer to the two excellent review articles $(2,3)$ from the pioneers of YY1 studies, Drs. Shi and Seto. Some other reviews also discussed the role of YY1 in cancer development (4$6)$. The focus of this article is the cancer-related function and regulation of YY1. The potential of YY1 as a therapeutic target of cancer therapy is also discussed.

\subsection{Discovery of YY1}

YY1 was initially discovered in 1991 by Shi et al as a transcriptional factor binding to the P5 promoter of adeno-associated virus (7). The inhibitory function of YY1 to this promoter can be converted to an activating effect upon association with a viral protein, E1A. By its very name, "Yin 
Yang" represents its two opposite capabilities of either repressing or stimulating gene transcription, and both functions have been demonstrated in numerous studies. In addition, YY1 can act as an initiator to direct and activate gene transcription (8). Two subsequent studies also reported subcloning of YY1 cDNA (NF-E1 and delta, respectively) and observed the regulatory role of YY1 in gene transcription. One of the studies demonstrated that YY1 is a binding protein of the negative-acting segment of the immunoglobulin kappa E3' enhancer (9). The other study showed that YY1 acts as a transcription activator of two genes coding ribosomal proteins rpL30 and rpL32, since the deletion of YY1 binding elements dramatically reduced their expression (10). In 1992, YY1 (named as UCRBP) was identified as a binding protein of the upstream conserved region in Moloney murine leukemia virus (MuLV) and negatively regulated the MuLV promoter activity (11).

\subsection{Expression and general function of YY1}

YY1 is ubiquitously expressed in all tissues and highly conserved among different species. Currently, YY1 cDNAs have been cloned from many species, including human (7), mouse $(10,12)$, rat (13), chicken (14), zebrafish (15) and xenopus (16). Drosophila has two orthologs of YY1, pleiohomeotic (pho) and pho like (phol) $(17,18)$, which have high degrees of similarity in the zinc finger regions with those of human YY1. The Seto group discovered a protein called YY2 that has $65 \%$ similarity in DNA sequence and $56 \%$ similarity in protein sequence to human YY1 (19). Due to the pronounced similarity at the zinc finger regions of these two proteins, YY2 binds to the same consensus sequence as YY1 but with much lower affinity (20). A latter report suggested that YY2 is a retroposed copy of YY1 that has been inserted into another gene locus (21).

A number of studies demonstrated the essential roles of YY1 in normal biological processes, such as embryonic development and gene imprinting. Donohoe et al attempted to generate YY1 knockout mice, but discovered that YY1 deletion is lethal to mouse embryonic development (22). The complex of YY1 and a chromatin isolator, CTCF, regulates the $\mathrm{X}$ chromosome binary switch by binding to a noncoding locus, Tsix that controls chromosome pairing and counting (23). Meanwhile, YY1 has been shown as a critical regulator of early B-cell development (24), genomic imprinting (25), and neuron development and differentiation $(26,27)$.
Affar et al presented a comprehensive study on the essential dosage-dependent functions of YY1 in embryonic development and cell cycle progression (28). They provided clear evidence that decreases of YY1 impair the growth and viability of mouse embryos in a dose-dependent manner. In addition, levels of YY1 tightly correlate with cell proliferation, and its depletion prevents cytokinesis and increases cellular sensitivity to various apoptotic stimuli.

\section{YY1 as a transcription factor}

The models of YY1-mediated gene repression and activation have been described in previous reviews $(2,3)$. To repress the expression of a gene, the association of YY1 with a target promoter may cause activator displacement, interference with the activator's function or recruitment of corepressors. When activating a target gene, YY1 may directly act as an activator, inhibit the activity of repressors or recruit coactivators.

As a member of the GLI-Krüppel class of zinc finger proteins, YY1 has been reported to regulate the expression of a large cohort of genes and/or promoters. The four zinc fingers at the C-terminal of YY1 (Figure 1) are responsible for binding to its target promoters. The YY1 binding motif was initially recognized as having two types of cores,

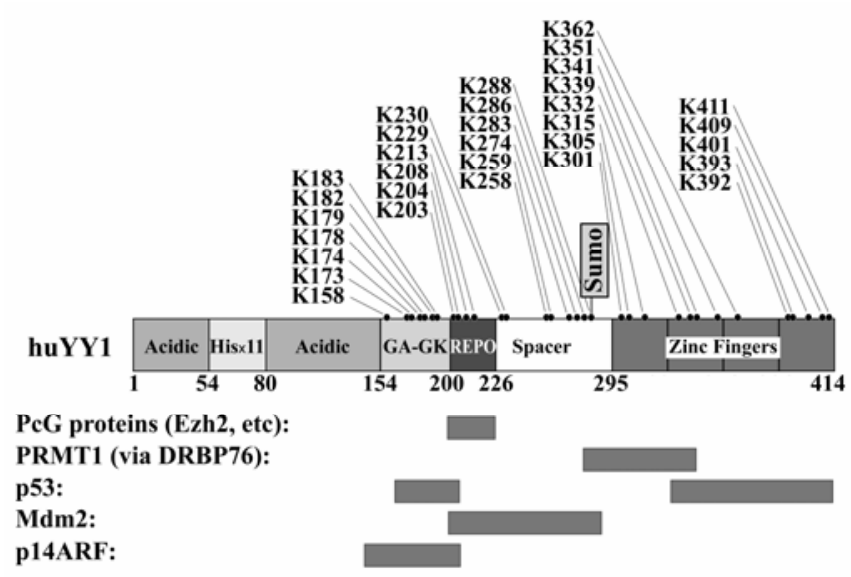

Figure 1. Domain structure of YY1 and interaction regions of histone methyltransferases and p53-related proteins. The YY1 sequence is based on the NCBI access number Z14077. All 32 lysine $(K)$ residues are labeled on the top and the SUMO conjugation site, K288, is indicated. The REPO motif of YY1 is necessary and sufficient in recruiting PcG proteins, such as Ezh2, to YY1-targeted promoters to promote histone $\mathrm{H} 3-\mathrm{K} 27$ methylation and establish gene silencing (68). The residues $261-333$ of $Y Y 1$ are responsible for recruiting PRMT1 via DRBP76 to methylate histone H3-R4 (65). The interactions domains of $\mathrm{p} 53, \mathrm{Mdm} 2$ and $\mathrm{p} 14 \mathrm{ARF}$ were mapped by in vitro protein binding experiments (47). For the binding domains of other YY1-interacting proteins, please refer to Seto's review (3). 
ACAT and CCAT (29). Recently, longer DNA binding motifs were also identified (30). In an earlier report on the prevalence of YY1 binding sites, over $7 \%$ of vertebrate genes and $24 \%$ of viral genes contained YY1 binding elements (31), suggesting a ubiquitous regulation of YY1 in different biological processes. Interestingly, although YY1 was first recognized as a transcription factor, one report indicated that the localization of YY1 is cell cycle-dependent (32). In this report, the authors presented that YY1 mainly stayed in cytoplasm at G1 phase, but translocated into the nucleus at the early and middle $\mathrm{S}$ phases and then moved back to the cytoplasm at late $\mathrm{S}$ phase. The entry of YY1 into the nucleus coincided with increased YY1-DNA association and DNA/histone synthesis, suggesting a regulatory role of YY1 in cell division and proliferation. The function of YY1-regulated genes covers cell growth, proliferation, cytokinesis, apoptosis, development and differentiation, indicating that YY1 plays an essential role in coordinating multiple biological pathways through a complex transcriptional network (28).

In this review, I have categorized these targets into two groups based on the regulatory consequence of YY1: activation and repression. Because this review focuses on the genes involved in tumorigenesis, each group has also been divided into three subgroups based on the functions of the targeted genes or promoters (Tables 1.1 and 1.2).

\subsection{YY1-activated gene expression}

Many YY1-activated genes exert oncogenic or proliferative effects on cells (Table 1.1). The regulation of YY1 on some of these genes is reminiscent of its originally observed "Yin Yang" effects on the P5 promoter of adenovirus (7). c-Myc is the first oncogene that was demonstrated to be activated by YY1 (33). In this study, YY1 was shown to increase the levels of two major c-Myc mRNA transcript variants. A recent report further delineated the mechanism underlying this regulation and revealed that the viral oncogenic protein E1A plays a role in YY1-mediated activation of c-Myc expression. E1A disassociates the YY1p300-HDAC3 complex, which initially inhibits c-Myc expression, and makes the c-Myc promoter more accessible by increasing regional histone acetylation (34). A similar scenario also occurs in the regulation of YY1 on another proto-oncogene, c-fos. Although YY1 can block c-fos gene expression through a direct interaction with the transcription complex ATF/CREB (35), this inhibition is also inverted by
E1A, which disrupts the ATF-CREB-YY1 complex and changes YY1 from a repressor to an activator of c-fos gene (36). Consistently, YY1 stimulates the association of serum response factor (SRF) with the c-fos serum response element (37), suggesting an activating function to c-fos gene expression. These studies indicate that YY1 does not activate, or may even repress, these proto-oncogenes in normal conditions. However, when stimulated by oncogenic signals, YY1 will facilitate tumorigenic processes.

Among the targets of YY1, several other genes also code oncogenic proteins. YY1 is highly expressed in breast cancer and stimulates the expression of ERBB2 $(38,39)$. ERBB2 (also known as Her2 and neu) is overexpressed in about $30 \%$ of breast cancers and generally correlated with a poor prognosis (40). Since overexpressed ERBB2 contributes to increased aggressiveness of cancer cells, it is a notable target of breast cancer therapy. Increased ERBB2 partly results from its gene amplification (41), which might put the physiological significance of its upregulation by YY1 in doubt. However, a recent study showed that YY1 protein levels are inversely correlated with ERBB2 gene amplification in breast tumors (39), suggesting that YY1 may play a role in ERBB2 upregulation when this gene is not amplified.

As a prosurvival endoplasmic reticulum (ER) chaperone, GRP78/BiP has been shown in multiple studies to possess oncogenic properties by stimulating tumor proliferation, survival, metastasis, and resistance to various therapies (42). Baumeister et al demonstrated that YY1 associates with the GRP78 promoter only in conditions of ER stress. YY1 acts as an essential coactivator of ATF6 and recruits histone $\mathrm{H} 4$ methyltransferase PRMT1 (Figure 1) to enhance GRP78 gene expression (43). This study revealed an important role of YY1 in regulating stress-induced modification of chromatin and promoting cell survival response to stress conditions.

Other oncogenic or proliferative genes activated by YY1 include COX-2 and OTX2 (Table 1.1). It is noteworthy that two studies suggested the contribution of YY1 to the epithelial-mesenchymal transition (EMT). EMT can be induced by multiple oncogenic pathways and is inhibited by the tumor invasion suppressor E-cadherin. A recent report demonstrates the regulation of YY1 to the transcription of Snail, a transcriptional repressor of E-cadherin (44). The association between YY1 and the $3^{\prime}$ enhancer of Snail is essential to Snail expression in melanoma cells, since decreased levels 
Table 1.1. YY1-activated genes/promoters linked to tumorigenesis.

\section{Gene/promoter}

Function of the gene product
References

A. Oncogenic, proliferative and/or overexpressed genes in cancer

C-Myc

ERBB2/Her2

COX-2

GRP78/BiP

OTX2

Snail

Msx2

Mitochondrial

genes: Cyto $C$ etc

DR- $\alpha$

VASAP-60/ PRKCSH/80K-H

\section{B. Tumor suppression genes}

ERGIC-53

HLJ1

p53

p73

Peg3 (via CSE2 binding element)

RIZ1
Transcription factor and oncogene of various cancers

Proto-oncogene in breast cancer

Oncogene of various cancers

Promoting tumor proliferation, survival, metastasis

and resistance to cancer therapies

Oncogene of medulloblastoma

Enhancing cell survival, movement and/or EMT

EMT and tumorigenesis

Cell respiration

Overexpressed in cancers

Elevated in breast cancer
$[33,139]$

$[33,39]$

[112]

$[42,43,140,141]$

$[142,143]$

$[44,144]$

$[45,145]$

[46]

$[146,147]$

[148]

Transmembrane lectin facilitating the efficient export of a

[99] subset of secretory glycoproteins from the endoplasmic reticulum; induced by ER-stress

Tumor and invasion suppressor

Tumor suppressor

A member of $\mathrm{p} 53$ family proteins

[52]

[149]

A histone methyltransferase

Altered expression in cancers, maybe a tumor suppressor

\section{Other regulatory proteins in tumorigenesis}

Epidermal growth factor receptor (EGFR)

Histone H3.2alpha

Histone $\mathrm{H} 4$

Line-1 (promoter)

Myelin Proteolipid protein (PLP)

OTK18

PARP-1

PCNA

$\mathrm{RE}-1$ silencing transcription factor (REST) or neuron-restrictive silencer factor (NRSF) gp91(phox)

B-type natriuretic peptide (BNP) Transferrin receptor (CD71)
Cell signaling molecules involved in diverse cellular

functions, including cell proliferation, differentiation, motility, and survival, and in tissue development

Aberrant modifications in cancers

Aberrant modifications in cancers

Showing altered methylation in cancers

Primary constituent of myelin in the central nervous system Induced by HIV infection

Promoting poly(ADP-ribosyl)ation; related to DNA damage repair

Involved in DNA synthesis and repair; cooperating with nucleophosmin/B23

Showing both tumor suppressor and oncogenic activities

Catalytic subunit of the NADPH oxidase; potential target of cancer therapy

Related to patients' response to cancer therapy

Related to poor prognosis and resistant to tamoxifen in breast cancer
[77] 
Table 1.2. YY1- repressed genes/promoters linked to tumorigenesis.

Gene/promoter

Function of the gene product
References

\section{A. Oncogenic and/or overexpressed genes}

c-fos

interferon $\beta$ (IFN- $\beta$ )

HOXB13

CREB

matrix metalloproteinase-9

(MMP-9)

steroidogenic acute regulatory (StAR)

\section{B. Tumor suppression genes}

microRNA-29

p21

p16(INK4a)

Peg3 and Usp29

(via CSE1 binding element)

$\mathrm{Rb}$

TGF-beta
Proto-oncogene

Potential target in cancer therapy

Promoting cancer progression

Transcription factor

Increasing expressed in various cancers

Related to some cancers, e.g. glial tumors
[162]

$[87,163]$

$[63,164]$

[69]

$[165,166]$
Tumor suppressor of rhabdomyosarcoma

Leading to cell cycle arrest

Tumor suppressor

Peg3: tumor suppression; Usp29: ubiquitin-specific protease 29

Tumor suppressor

Tumor suppressor
[57]

[55]

[56]

[149]

[54]

\section{Other regulatory proteins in tumorigenesis}

alpha3beta1-integrin
mu opioid receptor (MOR)
CD30
CXCR4
PPAR-delta
ERCC5/XPG
OX40
Cdk4
Cyclin D1
Involucrin
Hoxd4

alpha3beta1-integrin mu opioid receptor (MOR)

CXCR4

PPAR-delta

ERCC5/XPG

OX40

Cyclin D1

Hoxd4

$[117]$
$[102]$
$[167]$
$[168]$
$[169]$
$[170]$
$[171,172]$
$[55]$
$[55,173]$
$[174]$
$[175]$

of YY1 led to reduced Snail expression. Another group also reported that YY1 acts as a transcriptional activator of a homeobox gene, Msx2, which plays an important role in inducing EMT transition in different cell types (45).

In 2007, a comprehensive study by Cunningham and colleagues described the role of YY1 in mediating cell respiration through mitochondria (46). YY1-binding elements are highly enriched in mitochondrial genes, and siRNA-mediated YY1 depletion significantly reduces the expression of many mitochondrial genes and in turn decreases the oxygen consumption. Consistently, YY1 protein is required for the inhibition of the mitochondrial genes by rapamycin. Mechanistic studies suggested that the YY1-PGC-1a transcriptional complex is essential to the mitochondrial oxidative function, while mTOR interferes with this regulation through altering the YY1-PGC-1a complex. Overall, this study demonstrated that YY1 plays an essential role in maintaining the basal respiration of cells.

Although the research described above implicates a proliferative role of YY1, several studies also indicated that YY1 may promote genes with tumor suppression function. While most reports demonstrated that YY1 negatively regulates p53 at posttranslational level and inhibits p53 transcriptional activity (47-50), YY1 
exhibited stimulating effects in a study using a p53promoter reporter and overexpressed YY1, and this activation was further enhanced by cotransfected E1A (51), which seems to contradict the wellestablished oncogenic function of $\mathrm{E} 1 \mathrm{~A}$ protein. Another report also demonstrated the cooperative transcriptional activation of $\mathrm{p} 73$ by $\mathrm{YY} 1$ and $\mathrm{E} 2 \mathrm{~F} 1$ (52). Overexpressed YY1, together with activator protein 1 (AP-1), was shown to activate the transcription of HLJ1, a suppressor of tumor invasion (53).

\subsection{YY1-repressed gene expression}

YY1 represses several targets with potential tumor suppression function. YY1 exhibits an inhibitory effect on $\mathrm{Rb}$ expression. During myogenesis (muscle cell differentiation), the translocation of YY1 from the nucleus to the cytoplasm causes $\mathrm{Rb}$ gene activation, which leads to the exit of cell cycle and the consequent myogenesis (54). However, it is still unclear whether overexpressed YY1 during tumorigenesis can inactivate the $\mathrm{Rb}$ gene. One way in which YY1 antagonizes p53 function is through attenuating p53 target genes, including p21 (49). The same regulation was also observed in vascular smooth muscle cells (55). YY1 was reported to recruit HDAC3 and HDAC4 to the promoter of p16(INK4a) and repress its expression, which could release the cells from senescence (56). YY1, together with NFkappaB, could inhibit microRNA-29 (miR-29) (57), which is a potential tumor suppressor through activating $\mathrm{p} 53$.

Other YY1-regulated genes involved in tumorigenesis are shown in Tables 1.1 and 1.2. Based on the regulation of YY1 and the function of these genes, some discrepancies still exist, which makes it impossible to exclusively define the role of YY1 in cancer development. However, the tumorigenesis promoting effects of YY1 clearly override its function of tumor suppression. It is reasonable to hypothesize that the overall outcome of YY1-regulated processes depends on the oncogenic stimuli, cell types and the interplay with its recruited cofactors, whose availability may be altered at different physiological conditions.

\section{YY1 and protein post-translational modifications}

Posttranslational modifications have largely increased the complicity of the regulation on protein functions. Among several modifiable residues, lysines are the major targets of post-translational modifications, including acetylation, methylation, ubiquitination and sumoylation. The high-lysine (8\%) composition of YY1 has determined its liability as a target of multiple modifying groups. Interestingly, these lysine residues are mostly located in the middle region of the primary sequence of the protein, while none is among the first 157 residues (Figure 1) While YY1 itself is a target, it also regulates various protein modifications. A well established mechanism of YY1-regulated gene expression is through recruiting histone modifiers to the target promoters and modulating histone modifications. YY1 has been reported to interact with numerous proteins including many protein modifiers that promote acetylation, deacetylation, methylation, ubiquitination and sumoylation of histone or nonhistone proteins (Table 2).

\subsection{Acetylation}

The interaction of p300 and YY1 was initially demonstrated by Lee et al in 1995 (58). Interaction domain mapping experiments revealed that YY1 and E1A bind to distinct sites of $\mathrm{p} 300$, and the primary binding sites of $\mathrm{p} 300$ and E1A on YY1 do not overlap, suggesting that the three proteins potentially form a ternary complex. Hence, the molecular mechanism underlying the conversion of YY1 from a repressor to an activator by E1A is through recruiting p300 to acetylate histones on YY1 target promoters. One of the transcription-independent properties of YY1 is its inhibition of p300-mediated p53 acetylation (48). This is one of the multiple approaches employed by YY1 to antagonize p53 (Figure 2), since acetylation can both prevent p53 ubiquitination (59) and enhance p53 transcriptional activity by promoting p53-DNA association (60).

Another landmark study by Yang et al linked the regulation of YY1 to histone deacetylation (61). They discovered an YY1 binding protein, hRPD3, a homolog of yeast RPD3 that is the definition base of the Class I HDACs. This hRPD3, which was later determined to be HDAC2 (3), contributes to YY1repressed gene expression. Many other reports confirmed the importance of HDACs in YY1mediated gene repression. Luke et al revealed that YY1 interacts with a homeodomain protein, HoxA11, and recruits HDAC2 to HoxA11 target gene to abrogate HoxA11-mediated gene activation (62). Recently, YY1 was reported to recruit HDAC4 and repress the expression of HOXB13, which plays a role in growth arrest in androgen receptor-negative prostate cancer cells (63). 
Table 2. YY1 interacting proteins

Protein
Function

References

\section{A. Modifiers of histones and nonhistone proteins}

p300, CBP

HDAC1, 2, 3, 4, 5

Ezh1

Ezh2

PRMT1

Mdm2

Ubc9

PIASy
Acetylation of histone and nonhistone proteins

Deacetylation of histone and nonhistone proteins

Histone methyltransferease

Histone methyltransferease on H3-K27 and H1-K26

Histone methyltransferease on H4-R3

Ubiquitination of p53 and histones

E2 conjugating enzyme of sumoylation

E3 ligase of sumoylation
[58]

$[61,63,64,176,177]$

[66]

$[67,178]$

[65]

$[47,48]$

[72]

[72]

\section{B. Other chromatin remodeling proteins}

Nucleophosmin (NPM)/B23
CtBP1
RYBP
CTCF
INO80
SAP30

\section{Tumorigenesis and apoptosis}

p53

$\mathrm{Rb}$

p14ARF

E1A

Mdm2

c-Myc

c-Jun

Caspases ${ }^{*}$ 1, 3, 5, 6, 7

PARP-1

E2F2, E2F3

MBP1

$\alpha$-enolase

\section{General and other regulation}

RNA Pol. II, TFIIB, Sp1,

ATF/CREB, TAFII55

Notch1 receptor

SFMBT2

YY1AP (HCCA2)

YAF2

mTOR (FRAP1)

Raptor

PGC-1a

SHDAg and LHDAg

( $\sigma$-virus antigen)

Hoxa11

AP-2 (activator protein 2)

CP2

SMAD $1 / 4$
A histone chaperon involved in nucleosome formation

[79]

A corepressor involved in chromatin remodeling

A repressor present in PcG complex

Chromatin remodeling; its deregulation causes epigenetic

imbalance in cancer

Chromatin remodeling and DNA repair

Involved in LOH (Loss of Heterozygosity)
$[81,82]$

[83]

$[23,179]$

$[88,89]$

$[86,87]$
Tumor suppression and genome stability

Tumor suppression and genome stability

Tumor suppression

Oncogene leading to tumorigenesis

Oncogene enhancing p53 degradation

Oncogene transforming cells

Protooncogene

Proteases activated during apoptosis

Posttranslational modification, DNA repair

Regulating $\mathrm{Rb}$ pathway

c-Myc promoter binding protein 1 (MBP-1)

Tumor antigen in lung cancer
[47]

[91]

[47]

[7]

$[47,180]$

[181]

[94]

[95]

[76]

[84]

[139]

[139]

General gene transcription

[35,96-99]

Cell fate determination during embryonic development

$[139,182]$

[183]

Hepatocellular carcinoma-specific protein

[184-186]

Interacting with $\mathrm{MycN}$ in neuroblastoma

Key factor in transducing various stimuli to regulate a wide

$[187,188]$

range of cellular functions

Associate with mTOR, and regulate its expression and activity

A co-activator of $Y Y 1$ in regulating mitochondrial genes

[46]

Involved in TGF-beta and c-Jun-induced signaling cascade

[46]

[46]

[189]

Regulating uterine development

[62]

Acting as a co-factor to stimulate ERBB2 promoter

[38]

Transcription factor; interacting with the HXPR motif of YY1

[190]

TGF-beta signal pathway 
The transcriptional activity of $\mathrm{YY} 1$ is also regulated by acetylation (64). p300 and PCAF mediate the acetylation of the central region (residues 171-200) of YY1 and this modification augments YY1-mediated gene repression. The Cterminal of YY1 can also be acetylated by PCAF, which reduces the YY1-DNA association.

\subsection{Methylation}

As stated above, YY1 is an activator of c-Myc gene. Rezai-Zadeh et al demonstrated that the mechanism of this activation is through the YY1recruited PRMT1, a histone methyl-transferase specific to the arginine 3 of histone H4 (H4-R3) (65). The recruitment of PRMT1 by YY1 on the promoter of GRP78, a prosurvival ER chaperone, also leads to histone H4-R3 methylation and GRP78 gene activation (43). YY1 interacts with two lysinespecific histone methyl transferases, Ezh1 (enhancer of zeste homologue 1) and Ezh2, both of which mediate the methylation of histone H3-K27, a hallmark of gene silencing $(66,67)$. Caretti et al first demonstrated that YY1 is essential to Ezh2mediated methylation on histone $\mathrm{H} 3-\mathrm{K} 27$ in mouse skeletal muscle cells (67). This finding supports a regulatory role of YY1 in prostate cancer development, since the essential role of Ezh2 in prostate cancer development has been well established. Both YY1 and Ezh2 are overexpressed in various cancers. Thus, the recruitment of Ezh2 by YY1 may contribute to the aberrant epigenetic status of cancers. Transgenic studies in Drosophila indicated that the REPO (REcruitment of POlycomb proteins) motif, consisting of residues 201-226 of YY1 (Figure 1), is necessary and sufficient in recruiting Ezh2 and other polycomb group (PcG) proteins to establish transcriptional repression (68).

\subsection{Ubiquitination and sumoylation}

The turnover of YY1 is likely through ubiquitination and proteasomal degradation, since the treatment of a proteasome inhibitor led to accumulated YY1 protein (47). In neuronal cells, ubiquitinated YY1 interacts with $\mathrm{CtBP}$ and HDAC3 to establish a repressive complex (69). Meanwhile, we and others discovered that YY1 plays a role in regulating p53 ubiquitination and degradation $(47,48)$ (Figure 2). YY1 directly interacts with both p53 and Mdm2, a ubiquitin E3 ligase, and enhances the Mdm2-p53 interaction by forming a ternary complex with them. As a result, YY1 depletion leads to either apoptosis or cell cycle arrest, depending on the cell types $(28,47)$. Importantly, this regulation is

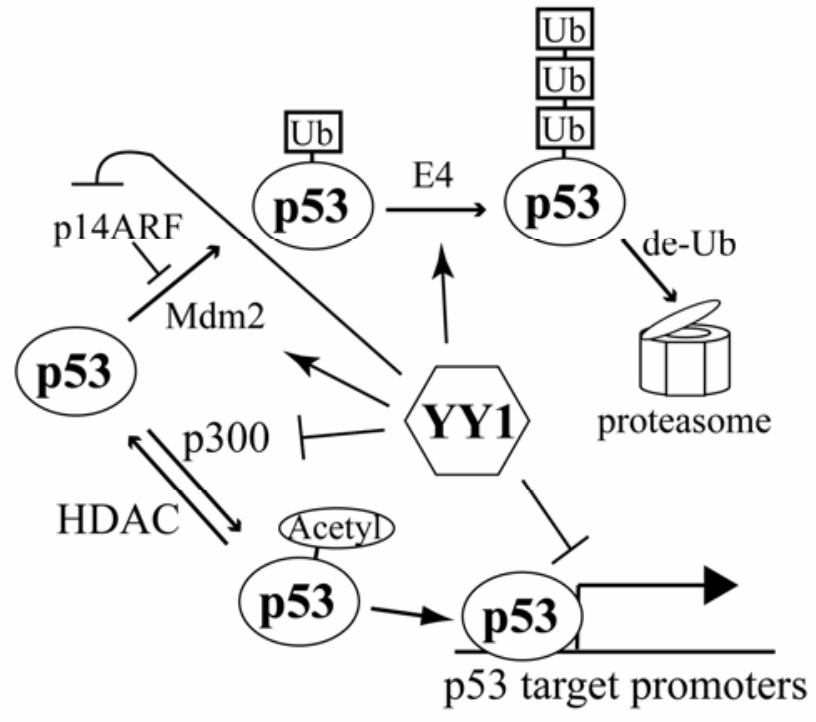

Figure 2. Schematic model of multiple mechanisms of YY1 to antagonize p53. YY1 inhibits p300-mediated p53 acetylation that endows p53 with increased stability and DNA binding affinity compared to deacetylated p53 [48]. YY1 also enhances Mdm2mediated p53 ubiquitination and polyubiquitination, and interferes with p14ARF-mediated p53 stabilization [47]. In addition, YY1 also inhibits the transcription of p53 target genes, such as p21. Acetyl: acetylation; Ub: ubiquitination; de-Ub: deubiquitination driven by ubiquitin-specific proteases.

independent to the transcriptional activity of YY1, since an YY1 mutant deficient in DNA binding retains the ability of stimulating p53 ubiquitination, and YY1 protein purified from a bacterial expression system is capable of enhancing p53 ubiquitination in vitro (47).

The functional outcomes of protein sumoylation and ubiquitination are very distinct (70). SUMO (Small Ubiquitin-related MOdifier) conjugations are normally present in specific regions responsible for protein-protein interactions. Therefore, sumoylation may alter the function of a protein by changing its binding partners (71). We have demonstrated that YY1 can be conjugated by SUMO-1, 2 and 3, and Lys288 of YY1 is the primary conjugating site (Figure 1) (72). YY1 sumoylation, which is stimulated by PIASy, a SUMO E3 ligase, negatively affects the transcriptional activity of YY1. In addition, we also revealed a direct interaction of YY1 with Ubc9, the only conjugating enzyme of protein sumoylation. The interaction of YY1 with Ubc9 and PIASy suggests a potential role of YY1 in regulating the sumoylation of its interacting partners.

The interaction of YY1 with different transcription factors and protein modifiers also contributes to YY1-mediated gene expression. As 
schematically shown in Figure 3, multiple studies demonstrated the regulation of YY1 to histone acetylation, deacetylation and methylation by p300, HDACs, Ezh2, Ezh1 and PRMT1, respectively. It is possible that these modifications occur on nonhistone proteins in an YY1-recruited transcriptional complex. In addition, Mdm2 and Ubc9 may also be recruited by YY1 to its targeted promoters and modify these cofactors to regulate gene expression.

\subsection{Other modifications}

YY1 is also a subject of other modifications. Some of the YY1 present in nucleus appears to be OGlcNAcylated regardless of the differentiation status of the cells, and glycosylated YY1 no longer interacts with Rb, although it still binds DNA (73). This study suggested that the glucose metabolism regulates YY1 protein by promoting its O-GlcNAcylation and consequently changing its activity. Although glycosylation frequently occurs to proteins expressed in cell membrane, it also plays an important role in regulating transcription. A number of transcription factors, such as Sp1 and RNA polymerase II, bear glycosylation that affects their transcriptional activity (74). Whether the glycosylation affects YY1mediated transcription, in addition to its interaction with $\mathrm{Rb}$, is still unclear. Moreover, YY1 interacts with poly (ADP-ribose) polymerase 1 (PARP1) and stimulates its function in catalyzing the synthesis of ADP-ribose polymers (75). Meanwhile, YY1 was reported to be transiently poly(ADP-ribosyl)ated after genotoxic treatment, which is coincides with the activation of PARP1 (76). Unlike glycosylation, the poly(ADP-ribosyl)ation decreased the DNAbinding affinity of YY1.

\section{YY1-interacted proteins and their function}

YY1 has been reported to interact with numerous proteins and most of these interactions are direct. One report suggested that YY1 may change its subcellular localization at different stages of the cell cycle (32). We observed that YY1 is predominantly localized in the nucleus but a small portion of YY1 also can be found in the cytoplasm, suggesting that its binding proteins are present in both compartments and YY1 possibly switches its binding partners during cell division and proliferation. The proteins that interact with YY1 can be categorized into four groups (Table 2), most of which possess regulatory function in tumorigenesis.

\subsection{Protein modifiers}

This has been discussed in Section 3 and summarized in Table 2.

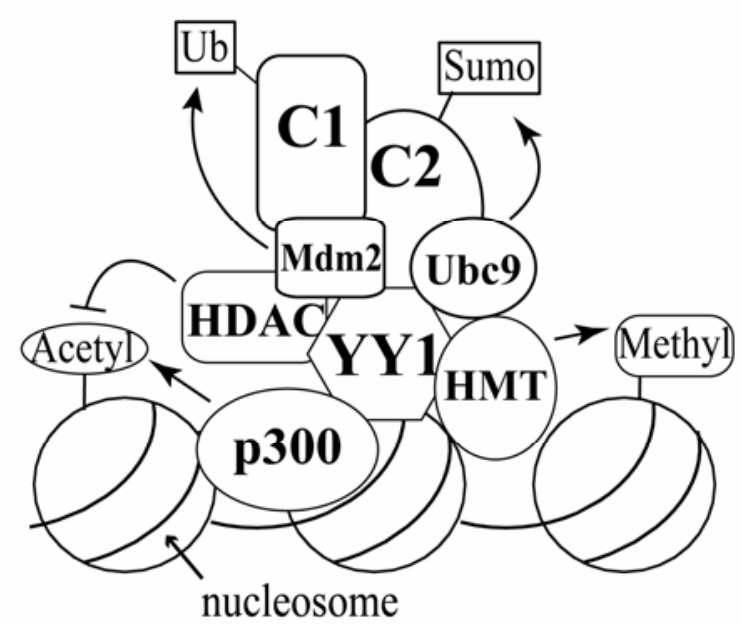

Figure 3. Schematic model of YY1-mediated protein modifications of histone and non-histone proteins on its target promoter. The interaction of $Y Y 1$ with protein modifiers may mediate the modifications of the components in an YY1-recruited transcriptional complex. YY1 recruits p300, HDAC and histone methyltransferase (HMT, such as Ezh2, Ezh1 and PRMT1) to acetylate, deacetylate and methylate histones, respectively. Function of other cofactors (designated as C1 and C2) may also be altered by YY1-mediated modifications, such as ubiquitination and sumoylation.

\subsection{Other chromatin remodeling proteins}

In addition to these protein modifiers that directly regulate histone modifications, a number of other YY1-interacting proteins are also involved in chromatin remodeling. YY1 directly regulates the gene expression of histone H3.2alpha and histone H4 $(77,78)$. These histone proteins are the primary components of the nucleosome. As a nucleolar protein and histone chaperone, nucleophosmin (NPM)/B23 regulates nucleosome formation. NPM forms a complex with YY1 both in vivo and in vitro. Interestingly, NPM relieves the transcriptional repression mediated by YY1 (79) and promotes the YY1-mediated activation of PCNA promoter (80). Among the polycomb group (PcG) proteins, YY1 is the only member that is known to directly and specifically bind DNA and recruit other PcG proteins to establish gene silencing. Using Drosophila as a model, the Atchison group demonstrated that the gene repression mediated by YY1 and other PcG proteins requires the co-repressor $\mathrm{CtBP}(81,82)$. A recent study in rat hippocampal cells indicated that CtBP specifically interacts with mono-ubiquitinated YY1 to repress matrix metalloproteinase-9 (MMP-9) gene transcription (69). Therefore, YY1 deubiquitination resulted from neuronal depolarization disrupts the repressive complex of 
YY1-CtBP-HDAC3 and in turn activates MMP-9 expression.

RYBP (Ring1- and YY1-binding protein) was initially identified as a corepressor in PcG complex (83). A later study indicated that RYBP mediates the interaction of YY1 with E2F protein, which leads to activation of the Cdc6 gene (84). At the protein level, RYBP antagonizes Mdm2-mediated p53 ubiquitination (85), in opposition to the effects of YY1.

The interaction between YY1 and SAP30, a component of the human histone deacetylase complex, has been proposed as an alternative mechanism of YY1-mediated gene repression (86). SAP30 promotes the recruitment of HDAC1 by YY1 to repress gene expression. In addition, the recruitment of the $\mathrm{Sin} 3 \mathrm{~A} / \mathrm{NCoR} / \mathrm{HDACs}$ repressor complex by YY1 inhibits the expression of interferon beta (IFN-beta) gene (87).

INO80 is a subfamily of SWI2/SNF2 chromatin remodeling proteins and plays regulatory roles in gene transcription, DNA repair and DNA replication. Two recent studies demonstrated the functional interplay between YY1 and INO80. When YY1 activates transcription of its target genes, INO80 acts as an essential co-activator and helps YY1 to gain access to the target promoters (88). In addition, YY1 and INO80 are essential to homologous recombination-based DNA repair and therefore may regulate the cellular response to genotoxic stress (89).

\subsection{Proteins involved in tumor suppression, oncogenesis, apoptosis and DNA damage}

Besides p53 and Mdm2, YY1 also interacts with many other proteins that directly regulate tumorigenesis. YY1 binds to tumor suppressor retinoblastoma $(\mathrm{Rb})$ in vitro. Either glycosylation of YY1 or phosphorylation of $\mathrm{Rb}$ disrupts this interaction $(73,90)$. In cell-based experiments, only hypophosphorylated Rb interacts with YY1 and this interaction disrupts the YY1-DNA association (90), suggesting that $\mathrm{Rb}$ may be involved in YY1mediated transcriptional regulation. Interestingly, the YY1-Rb complex was only observed in resting cells, but not in serum or lipopolysaccharide (LPS) stimulated cells $(90,91)$, indicating their interaction is cell cycle-regulated. Another YY1-interacting tumor suppressor is p14ARF. Our functional study demonstrated that YY1 competes with p14ARF and therefore attenuates p14ARF-mediated p53 activation (47), which is another way in which YY1 antagonizes p53 function (Figure 2).
YY1 also interacts with many oncogene products, including E1A, c-Myc, c-Jun and Mdm2. As described above, viral oncogene E1A converts YY1 from a transcriptional repressor to an activator. As an YY1 interacting protein, c-Myc prevents YY1 from associating with its cofactors, but does not block its binding to DNA (92). YY1-c-Myc interaction mediates the stimulation of Surf-1 in the MAP kinase cascade (93). Similarly, c-Jun also interacts with YY1 and decreases the binding affinity of YY1 to its consensus binding element (94).

Krippner-Heidenreich et al provided direct evidence of the involvement of YY1 in apoptosis (95). Various apoptotic stimuli could promote rapid translocation of YY1 into cell nucleus and lead to cleavage of YY1 at Asp12-Gly and Asp119-Gly. Interestingly, one of these N-terminal truncated forms of YY1 could enhance Fas-induced apoptosis, suggesting YY1 plays a role in positive feedback during apoptosis. An in vitro study showed that YY1 was cleaved by caspase 1, 3, 5, 6 and 7 (95).

\subsection{Other regulatory proteins}

YY1 interacts with many general transcriptional factors and cofactors, such as RNA polymerase II, ATF/CREB, and Sp1 (35,96-99). This suggests that YY1 not only recruits other transcriptional cofactors to its target promoters, but also potentially acts as a cofactor recruited by others. Importantly, the presence of YY1 in a transcriptional complex creates an interface for these YY1-interacted protein modifiers that may alter the function of transcriptional machinery by modulating their posttranslational modifications (Figure 3). The interactions of YY1 with these general transcriptional factors and the regulatory proteins in Notch, TGF-beta, mTOR signaling pathways, as well as cell respiration (Table 2), once again indicate a critical role of YY1 in different biological processes.

\section{YY1 being regulated}

Although most reports have described how YY1 regulates the expression or modifications of other genes or proteins, some studies also demonstrated how YY1 expression and function are regulated (Table 3).

YY1 expression was shown to be upregulated by two growth factors, insulin-like growth factor-1 (IGF-1) and fibroblast growth factor-2 (FGF-2) $(100,101)$. YY1 exhibited low or absent expression in NIH3T3 cells that were quiescent or cultured in serum-deprived medium. Consistently, YY1 expression could be equally stimulated by serum or 
Table 3. Factors that regulate the function of YY1

Factor

Insulin-like growth factor-1 (IGF-1)

Fibroblast growth factor-2 (FGF-2)

TNF-alpha/NF-kappaB

Morphine

Lysophosphatidylcholine

(lysoPC)

C/EBP-beta

Prohibitin

MicroRNA-29

DETANONOate (nitric oxide donor)

Staphylococcal enterotoxin A (SEA)

Naloxone

RKIP

Rituximab

Lipopolysaccharide (LPS)

Sumoylation/PIASy

Ubiquitination

Different apoptotic stimuli and DNA synthesis inhibitor

Myeloid nuclear differentiation

antigen (MNDA)
Effect on YY1

References
IGF-1 (100). Injury of smooth muscle cells also led to an increase of YY1 expression; this stimulation was abolished by treatment with FGF-2 antibody (101). Proliferative drugs, such as morphine and lysophosphatidylcholine, also increased YY1 expression $(102,103)$.

YY1 expression is stimulated by transcription factor NF-kappaB that directly binds to YY1 promoter through its subunit p50/p65 heterodimer (104). Consistently, PC-3 cells treated by TNF-alpha exhibited elevated levels and increased DNAbinding activity of YY1. On the other hand, genetic ablation of the p65 subunit of NF-kappaB in both cultured cells and adult skeletal muscle correlated with reduced YY1 transcripts and protein (105). A chimeric antibody against CD20, rituximab, which inhibits constitutive NF-kappaB activity and therefore sensitizes tumor cells in B cell nonHodgkin's lymphoma and leukemias, represses YY1 expression (106).

Several mechanisms have been reported to downregulate YY1 expression. At the transcriptional level, YY1 expression is inhibited by prohibitin through E2F1 binding sites (107). While YY1 negatively regulates miR-29 expression, miR-29 also targets the 3'-UTR of YY1 mRNA and blocks its translation (57). The interplay between YY1 and miR-29 implicates their function in skeletal myogenesis and rhabdomyosarcoma development.

As a metastasis suppressor gene, Raf kinase inhibitor protein (RKIP) is poorly expressed in cancers. RKIP downregulates YY1 expression through inhibiting its transcription (108). Hence, RKIP overexpression increases tumor cell sensitivity to TRAIL via blocking YY1 expression. YY1 expression can also be inhibited by DETANONOate (a nitric oxide donor), naloxone (a drug used to counter the effects of opioid overdose) and staphylococcal enterotoxin (102,109-111).

In addition to regulating YY1 expression, some factors also affect YY1 function. Both lipopolysaccharide (LPS) and myeloid nuclear differentiation antigen (MNDA) enhance YY1 binding affinity to its target promoters $(112,113)$. Mono-ubiquitinated YY1 recruits CtBP and HDAC3 to repress Mmp-9 gene expression (69), while PIASymediated sumoylation inhibits YY1-mediated gene transcription (72). Different apoptotic stimuli, including the DNA synthesis inhibitor aphidicolin, can translocate and cleave YY1 protein $(32,95)$. 
Overall, YY1 expression is stimulated by proliferative stimuli, while antiproliferative signals tend to antagonize YY1.

\section{YY1 expression in cancers and its correlation with disease outcome}

Most processes mediated by YY1 are cancerrelated, while YY1 itself is also differentially regulated during apoptosis and tumorigenesis. Therefore, the studies summarized above provide unequivocal evidence for the essential role of YY1 in tumorigenesis. The regulatory role of YY1 in various signaling pathways may explain its aberrant expression in cancers. YY1 overexpression has been demonstrated in human breast cancer (38), prostate carcinoma (114), acute myeloid leukemia (115), osteosarcoma $(116,117)$, cervical cancer (118), brain cancer (119), ovarian cancer (120), large B-cell and follicular lymphoma (121) and colon cancer (122). Currently, the mechanisms of YY1 increase in these cancers remain unclear. As discussed in Section 5, YY1 expression can be regulated by multiple pathways and its upregulation can be achieved by different proliferative stimuli. Therefore, it is unlikely that a universal mechanism can be applied to YY1 upregulation in all cancers.

In prostate cancer, elevated YY1 expression correlates with higher morphologic grades or malignant histological phenotypes (114). Similarly, YY1 overexpression in osteosarcoma is positively and strongly correlated with the degrees of malignancy $(116,123)$. Consistently, YY1 is also upregulated in metastatic breast cancer (124) and its staining intensity in colon cancer is more pronounced in poorly differentiated tumors than in moderately or well-differentiated colon cancers (122).

As discussed above, the overall effect of YY1 on cells is proliferative or oncogenic, and YY1 overexpression is prevalent in most cancers. However, the expression of YY1 does not show a defined correlation with the susceptibility of cancer reoccurrence or length of patients' survival. In prostate, colon and ovarian cancers, YY1 expression positively correlated with the long-term survival periods of patients $(114,120,122,125)$. In contrast, among patients with large B-cell and follicular lymphoma, high levels of YY1 were associated with poor outcome, including a shorter survival interval (121). The mechanisms of this apparent paradox remain unknown. A recent study by Matsumura et al investigated the positive correlation between YY1 expression and the length of survival in patients with ovarian cancer (125). Their results suggested that the overexpression of $\mathrm{YY} 1$ and E2F could sensitize ovarian cancer cells to the treatment of taxane, a class of drugs that disrupt the function of microtubules. As a result, patients with higher expression of YY1 exhibited better response to the therapy and therefore had longer survival.

Despite the fact that YY1 exhibits oncogenic function in cancer development, an in vitro study using colon cancer cells showed no evidence of gene amplification or chromosomal translocation of YY1 (122). However, two YY1 mRNA isoforms (7.5 and $2.9 \mathrm{~kb})$ were substantially overexpressed and aneuploidy was also observed.

\section{The role of YY1 in tumorigenesis and its potential as a target in cancer therapy}

The essential regulation of YY1 in numerous cancer-related pathways and its increased expression in various cancers strongly implicate the importance of YY1 in cancer development and progression.

As a guardian of the genome, the tumor suppressor p53 plays a vital role in preventing malignant transformation of cells. Consistently, p53 mutations have been reported in over $50 \%$ of cancers. In vitro and in vivo studies also indicated that $\mathrm{p} 53$ inactivation can immortalize the cells and lead to deregulated cell proliferation or tumor formation. As described above, YY1 antagonizes p53 through several mechanisms (Figure 2), including enhancing p53 ubiquitination and degradation (47), blocking p53 acetylation (48), attenuating p14ARF-mediated p53 stabilization (47), and inhibiting p53-mediated transcription (49). These multiple and consistently negative effects of YY1 on p53 stability and function implicate p53 as a primary target of overexpressed YY1 in cancer cells and strongly suggest an oncogenic or proliferative role of YY1 in cancer development.

We hypothesize that YY1 coordinately regulates the function of Ezh2 and p53 to facilitate cancer development. Ezh2 has been identified as a bona fide oncogene (126) and used as a marker of cancers with aggressive and metastatic potential. Functionally, Ezh2 is essential to cancer progression and invasion $(127,128)$, and its overexpression increases the likelihood of therapy failure (129). In prostate cancer, although p53 deficiency did not lead to prostate carcinoma in a mouse model, p53 deletion augments the cancer development in Pten null mice (130). Our proposed model is that the regulation of p53 and Ezh2 by YY1 may take place at different stages of cancer development (Figure 4). 
Firstly, since nearly half of the cancers retain functional p53, especially at early stages, a developing phase must exist in the primary malignancy to defeat the genomic surveillance or tumor suppression function of p53 prior to its inactivation. We hypothesize that at this earlier stage, highly expressed YY1 plays a role to antagonize p53 function, which consequently initiates and/or promotes cell malignancy. Meanwhile, the suppression of Ezh2 expression by p53 (131) will also be released by YY1-mediated p53 inactivation and this in turn leads to increased levels of Ezh2 (Figure 4). Secondly, Ezh2 increase is always linked to tumor progression, invasion and metastasis (132-134), which are the later events of cancer development. We hypothesize that elevated YY1 expression is essential for Ezh2 to exert its methyltransferase activity and establish aberrant epigenetics, which augments cancer progression. At this stage, most of the cancers may already have acquired p53 mutations, which will augment the cancer progression. To these retaining functional p53, YY1 may still play a role in attenuating its function.

Noteworthily, our model suggests that YY1 antagonizes the functional p53 during the cancer development. This hypothesis does not conflict with the Vogelstein model for colorectal tumorigenesis $(135,136)$, which proposed that the acquired p53 inactivation, mostly through gene mutations, is a frequent event at the late stages of cancers. Based on the published data, YY1 is likely an important negative regulator of p53 throughout the whole malignant process, especially in the cancers preserving functional p53 proteins. Certainly, we cannot exclude the possibility that the contribution of our model to tumorigenesis is more important in certain types of cancers than the others.

Several studies described the potential of YY1 as an effective target in cancer therapy. The Bonavida group extensively studied the role of YY1 in chemoand immuno-resistance in cancer therapy and concluded that YY1 levels could be used to predict the therapeutic responsiveness (137). Since YY1 negatively regulates the expression of Fas, the inhibition of YY1 by nitric oxide or rituximab could upregulate Fas and sensitize the tumor cells to Fasinduced apoptosis $(110,138)$. In addition, YY1 also inhibits the expression of DR5, a death receptor mediating the extrinsic pathway of apoptosis. Therefore, Raf-1 kinase inhibitor protein (RKIP) could decrease YY1 expression and consequently upregulate DR5 to sensitize the cells to TRAIL-

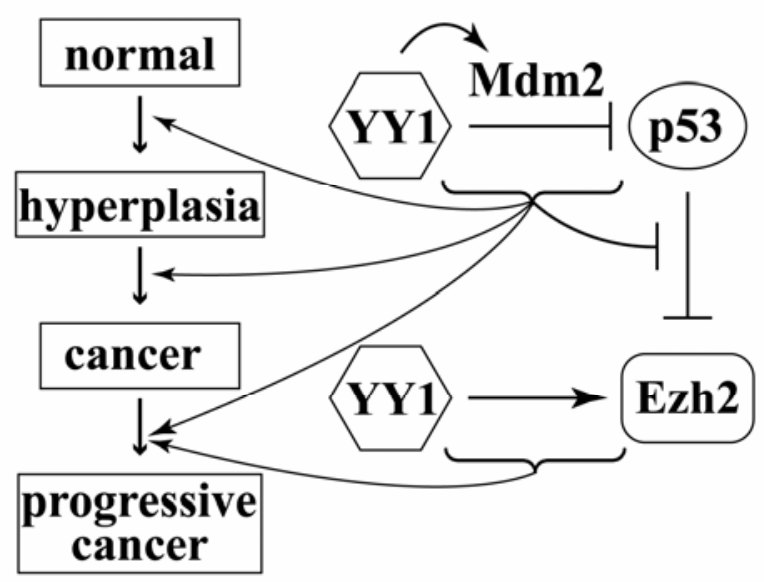

Figure 4. Schematic model of the regulation of p53 and Ezh2 by YY1 in cancer development. YY1 promotes Mdm2-mediated p53 degradation $[47,48,55]$ and inhibits its transcriptional activity [49]. This leads to tumor initiation and cancer development. Moreover, the suppression of p53 on Ezh2 gene expression [131] will also be released by YY1's antagonism to p53, which results in increased Ezh2 expression. At the late stage of cancer, YY1 recruits Ezh2 for histone methylation and consequently promotes tumor progression. Meanwhile, overexpressed $Y Y 1$ may also inhibit p53 activities in cancers retaining functional p53 proteins.

induced apoptosis (108).

A recent report by de Nigris et al focused on the role of YY1 in cell invasion, angiogenesis and metastasis (123). Their study demonstrated that YY1 depletion significantly decreased cell invasion and metastasis growth, which was associated with reduced endothelial growth factor (VEGF) and angiogenesis. This finding clearly suggests that YY1 is a promising and effective target in the therapy of bone cancer.

As summarized above, although discrepancies still exist, the overall function of YY1 is understood as both inhibiting tumor suppression processes and promoting oncogenic events. Especially, YY1 is an essential regulatory factor of numerous epigenetic events and its expression may affect many different biological processes leading to tumorigenesis. Theoretically, simultaneously targeting several pathways related to cancer development should result in more efficient and prompt outcome than targeting each of them individually. If a regulatory protein contributing to the abnormality of several processes toward malignancy can be identified, targeting this key regulator may exhibit a substantial impact by reversing or adjusting 
multiple pathways concurrently. Given its unique properties in mediating multiple epigenetic events and its causal links with various cancers, YY1 is likely one of these key regulatory proteins in cancer development and therefore can serve as an effective target in therapeutic treatment of cancers. Thus, targeting or adjusting YY1 in cancer therapy can potentially reverse the aberrant epigenetics of cancer cells and restore their normality. This will be especially important to the cancers in critical organs where radical surgery is not applicable.

\section{Acknowledgement}

The author thanks Kazushi Inoue, Karen Klein and Huifei Liu for critical reading and helpful discussions in the preparation of this article. The support for the work came from grants of the Department of Defense (PC060490) and American Cancer Society (RSG-09-082-01-MGO) to the author.

\section{Conflicts of Interest}

No potential conflicts of interest to disclose.

\section{References}

1. Hanahan D, Weinberg RA. The hallmarks of cancer. Cell 2000;100:57-70.

2. Shi Y, Lee JS, Galvin KM. Everything you have ever wanted to know about Yin Yang 1..... Biochim Biophys Acta 1997;1332:F49-66.

3. Thomas MJ, Seto E. Unlocking the mechanisms of transcription factor YY1: are chromatin modifying enzymes the key? Gene 1999;236:197-208.

4. Gordon S, Akopyan G, Garban H, Bonavida B. Transcription factor YY1: structure, function, and therapeutic implications in cancer biology. Oncogene 2006;25:1125-42.

5. Wang CC, Chen JJ, Yang PC. Multifunctional transcription factor YY1: a therapeutic target in human cancer? Expert Opin Ther Targets 2006;10:253-66.

6. Castellano G, Torrisi E, Ligresti G, et al. The involvement of the transcription factor Yin Yang 1 in cancer development and progression. Cell Cycle 2009;8:1367-72.

7. Shi Y, Seto E, Chang LS, Shenk T. Transcriptional repression by YY1, a human GLI-Kruppel-related protein, and relief of repression by adenovirus E1A protein. Cell 1991;67:377-88.

8. Seto E, Shi Y, Shenk T. YY1 is an initiator sequencebinding protein that directs and activates transcription in vitro. Nature 1991;354:241-5.

9. Park K, Atchison ML. Isolation of a candidate repressor/activator, NF-E1 (YY-1, delta), that binds to the immunoglobulin kappa 3 ' enhancer and the immunoglobulin heavy-chain mu E1 site. Proc Natl Acad Sci USA 1991;88:9804-8.

10. Hariharan N, Kelley DE, Perry RP. Delta, a transcription factor that binds to downstream elements in several polymerase II promoters, is a functionally versatile zinc finger protein. Proc Natl Acad Sci U S A 1991;88:9799-803.

11. Flanagan JR, Becker KG, Ennist DL, et al. Cloning of a negative transcription factor that binds to the upstream conserved region of Moloney murine leukemia virus. Mol Cell Biol 1992;12:38-44.

12. Safrany G, Perry RP. Characterization of the mouse gene that encodes the delta/YY1/NF-E1/UCRBP transcription factor. Proc Natl Acad Sci USA 1993;90:5559-63.

13. Chen KS, Paladugu A, Aldaz CM, Gould MN. Cloning and chromosomal localization of the rat Stat5 and Yy1 genes. Cytogenet Cell Genet 1996;74:277-80.

14. Bernard M, Voisin P. Photoreceptor-specific expression, light-dependent localization, and transcriptional targets of the zinc-finger protein Yin Yang 1 in the chicken retina. $J$ Neurochem 2008;105:595-604.

15. Song HD, Sun XJ, Deng M, et al. Hematopoietic gene expression profile in zebrafish kidney marrow. Proc Natl Acad Sci USA 2004;101:16240-5.

16. Pisaneschi G, Ceccotti S, Falchetti ML, Fiumicino S, Carnevali F, Beccari E. Characterization of FIII/YY1, a Xenopus laevis conserved zinc-finger protein binding to the first exon of L1 and L14 ribosomal protein genes. Biochem Biophys Res Commun 1994;205:1236-42.

17. Brown JL, Fritsch C, Mueller J, Kassis JA. The Drosophila pho-like gene encodes a YY1-related DNA binding protein that is redundant with pleiohomeotic in homeotic gene silencing. Development 2003;130:285-94.

18. Brown JL, Mucci D, Whiteley M, Dirksen ML, Kassis JA. The Drosophila Polycomb group gene pleiohomeotic encodes a DNA binding protein with homology to the transcription factor YY1. Mol Cell 1998;1:1057-64.

19. Nguyen N, Zhang X, Olashaw N, Seto E. Molecular cloning and functional characterization of the transcription factor YY2. J Biol Chem 2004;15:15.

20. Kim JD, Faulk C, Kim J. Retroposition and evolution of the DNA-binding motifs of YY1, YY2 and REX1. Nucleic Acids Res 2007;35:3442-52.

21. Luo C, Lu X, Stubbs L, Kim J. Rapid evolution of a recently retroposed transcription factor YY2 in mammalian genomes. Genomics 2006;87:348-55.

22. Donohoe ME, Zhang X, McGinnis L, Biggers J, Li E, Shi Y. Targeted disruption of mouse Yin Yang 1 transcription factor results in peri-implantation lethality. Mol Cell Biol 1999;19:7237-44.

23. Donohoe ME, Zhang LF, Xu N, Shi Y, Lee JT. Identification of a ctcf cofactor, yy1, for the $\mathrm{x}$ chromosome binary switch. Mol Cell 2007;25:43-56.

24. Liu H, Schmidt-Supprian M, Shi Y, et al. Yin Yang 1 is a critical regulator of B-cell development. Genes Dev 2007;21:1179-89.

25. Kim J, Kim JD. In vivo YY1 knockdown effects on genomic imprinting. Hum Mol Genet 2008;17:391-401. 
26. He Y, Dupree J, Wang J, et al. The transcription factor yin yang 1 is essential for oligodendrocyte progenitor differentiation. Neuron 2007;55:217-30.

27. He Y, Casaccia-Bonnefil P. The Yin and Yang of YY1 in the nervous system. J Neurochem 2008;106(4):1493-502. 28. Affar el B, Gay F, Shi Y, et al. Essential dosagedependent functions of the transcription factor yin yang 1 in late embryonic development and cell cycle progression. Mol Cell Biol 2006;26:3565-81.

29. Yant SR, Zhu W, Millinoff D, Slightom JL, Goodman M, Gumucio DL. High affinity YY1 binding motifs: identification of two core types (ACAT and CCAT) and distribution of potential binding sites within the human beta globin cluster. Nucleic Acids Res 1995;23:4353-62.

30. Kim J, Kim J. YY1's longer DNA-binding motifs. Genomics 2009;93:152-8.

31. Hyde-DeRuyscher RP, Jennings E, Shenk T. DNA binding sites for the transcriptional activator/repressor YY1. Nucleic Acids Res 1995;23:4457-65.

32. Palko L, Bass HW, Beyrouthy MJ, Hurt MM. The Yin Yang-1 (YY1) protein undergoes a DNA-replicationassociated switch in localization from the cytoplasm to the nucleus at the onset of S phase. J Cell Sci $2004 ; 117(\mathrm{Pt}$ 3):465-76.

33. Riggs KJ, Saleque S, Wong KK, et al. Yin-yang 1 activates the c-myc promoter. Mol Cell Biol 1993;13:748795.

34. Kadeppagari RK, Sankar N, Thimmapaya B. Adenovirus transforming protein E1A induces c-Myc in quiescent cells by a novel mechanism. J Virol 2009;83:4810-22.

35. Zhou Q, Gedrich RW, Engel DA. Transcriptional repression of the c-fos gene by $\mathrm{YY} 1$ is mediated by a direct interaction with ATF/CREB. J Virol 1995;69:4323-30.

36. Zhou Q, Engel DA. Adenovirus E1A243 disrupts the ATF/CREB-YY1 complex at the mouse c-fos promoter. J Virol 1995;69:7402-9.

37. Natesan S, Gilman M. YY1 facilitates the association of serum response factor with the c-fos serum response element. Mol Cell Biol 1995;15:5975-82.

38. Begon DY, Delacroix L, Vernimmen D, Jackers P, Winkler R. Yin Yang 1 cooperates with activator protein 2 to stimulate ERBB2 gene expression in mammary cancer cells. J Biol Chem 2005;280:24428-34.

39. Allouche A, Nolens G, Tancredi A, et al. The combined immunodetection of AP-2alpha and YY1 transcription factors is associated with ERBB2 gene overexpression in primary breast tumors. Breast Cancer Res 2008;10:R9.

40. Harari D, Yarden Y. Molecular mechanisms underlying ErbB2/HER2 action in breast cancer. Oncogene 2000;19(53):6102-14.

41. Seshadri R, Matthews C, Dobrovic A, Horsfall DJ. The significance of oncogene amplification in primary breast cancer. Int J Cancer 1989;43:270-2.

42. Lee AS. GRP78 induction in cancer: therapeutic and prognostic implications. Cancer Res 2007;67(8):3496-9.

43. Baumeister P, Luo S, Skarnes WC, et al. Endoplasmic reticulum stress induction of the Grp78/BiP promoter: activating mechanisms mediated by YY1 and its interactive chromatin modifiers. Mol Cell Biol, 2005;25:4529-40.

44. Palmer MB, Majumder P, Cooper JC, Yoon H, Wade PA, Boss JM. Yin Yang 1 regulates the expression of Snail through a distal enhancer. Mol Cancer Res 2009;7:221-9.

45. Tan DP, Nonaka K, Nuckolls GH, et al. YY1 activates Msx2 gene independent of bone morphogenetic protein signaling. Nucleic Acids Res 2002;30:1213-23.

46. Cunningham JT, Rodgers JT, Arlow DH, Vazquez F, Mootha VK, Puigserver P. mTOR controls mitochondrial oxidative function through a YY1-PGC-1alpha transcriptional complex. Nature 2007;450:736-40.

47. Sui G, Affar el B, Shi Y, et al. Yin Yang 1 is a negative regulator of p53. Cell 2004;117:859-72.

48. Gronroos E, Terentiev AA, Punga T, Ericsson J. YY1 inhibits the activation of the p53 tumor suppressor in response to genotoxic stress. Proc Natl Acad Sci USA 2004;101:12165-70.

49. Yakovleva T, Kolesnikova L, Vukojevic V, et al. YY1 binding to a subset of p53 DNA-target sites regulates p53dependent transcription. Biochem Biophys Res Commun 2004;318:615-24.

50. Bain M, Sinclair J. Targeted inhibition of the transcription factor YY1 in an embryonal carcinoma cell line results in retarded cell growth, elevated levels of p53 but no increase in apoptotic cell death. Eur J Cell Biol 2005;84:543-53.

51. Furlong EE, Rein T, Martin F. YY1 and NF1 both activate the human p53 promoter by alternatively binding to a composite element, and YY1 and E1A cooperate to amplify p53 promoter activity. Mol Cell Biol 1996;16:593345.

52. Wu S, Murai S, Kataoka K, Miyagishi M. Yin Yang 1 induces transcriptional activity of $\mathrm{p} 73$ through cooperation with E2F1. Biochem Biophys Res Commun 2008;365:75-81. 53. Wang CC, Tsai MF, Dai TH, et al. Synergistic activation of the tumor suppressor, HLJ1, by the transcription factors YY1 and activator protein 1. Cancer Res 2007;67:4816-26.

54. Delehouzee S, Yoshikawa T, Sawa C, et al. GABP, HCF-1 and YY1 are involved in Rb gene expression during myogenesis. Genes Cells 2005;10:717-31.

55. Santiago FS, Ishii H, Shafi S, et al. Yin Yang-1 inhibits vascular smooth muscle cell growth and intimal thickening by repressing $\mathrm{p} 21 \mathrm{WAF} 1 / \mathrm{Cip} 1$ transcription and p21WAF1/Cip1-Cdk4-cyclin D1 assembly. Circ Res 2007;101:146-55.

56. Wang X, Feng Y, Xu L, et al. YY1 restrained cell senescence through repressing the transcription of $\mathrm{p} 16$. Biochim Biophys Acta 2008;1783:1876-83.

57. Wang H, Garzon R, Sun H, et al. NF-kappaB-YY1miR-29 regulatory circuitry in skeletal myogenesis and rhabdomyosarcoma. Cancer Cell 2008;14:369-81.

58. Lee JS, Galvin KM, See RH, et al. Relief of YY1 transcriptional repression by adenovirus E1A is mediated by E1A-associated protein p300. Genes Dev 1995;9:118898. 
59. Li M, Luo J, Brooks CL, Gu W. Acetylation of p53 inhibits its ubiquitination by Mdm2. J Biol Chem 2002;277:50607-11.

60. Luo J, Li M, Tang Y, Laszkowska M, Roeder RG, Gu W. Acetylation of p53 augments its site-specific DNA binding both in vitro and in vivo. Proc Natl Acad Sci USA 2004;101:2259-64.

61. Yang WM, Inouye C, Zeng Y, Bearss D, Seto E. Transcriptional repression by YY1 is mediated by interaction with a mammalian homolog of the yeast global regulator RPD3. Proc Natl Acad Sci USA 1996;93:1284550 .

62. Luke MP, Sui G, Liu H, Shi Y. Yin Yang 1 physically interacts with HOXA11 and represses HOXA11-dependent transcription. J Biol Chem 2006;281:33226-32

63. Ren G, Zhang G, Dong Z, et al. Recruitment of HDAC4 by transcription factor YY1 represses HOXB13 to affect cell growth in AR-negative prostate cancers. Int $\mathrm{J}$ Biochem Cell Biol 2009;41:1094-101.

64. Yao YL, Yang WM, Seto E. Regulation of transcription factor YY1 by acetylation and deacetylation. Mol Cell Biol 2001;21:5979-91.

65. Rezai-Zadeh N, Zhang X, Namour F, et al. Targeted recruitment of a histone $\mathrm{H} 4$-specific methyltransferase by the transcription factor YY1. Genes Dev 2003;17:1019-29.

66. Wang L, Brown JL, Cao R, Zhang Y, Kassis JA, Jones RS. Hierarchical recruitment of polycomb group silencing complexes. Mol Cell, 2004;14:637-46.

67. Caretti G, Di Padova M, Micales B, Lyons GE, Sartorelli V. The Polycomb Ezh2 methyltransferase regulates muscle gene expression and skeletal muscle differentiation. Genes Dev 2004;18:2627-38.

68. Wilkinson FH, Park K, Atchison ML. Polycomb recruitment to DNA in vivo by the YY1 REPO domain. Proc Natl Acad Sci USA 2006;103:19296-301.

69. Rylski M, Amborska R, Zybura K, et al. Yin Yang 1 is a critical repressor of matrix metalloproteinase-9 expression in brain neurons. J Biol Chem 2008;283:3514053.

70. Gill G. SUMO and ubiquitin in the nucleus: different functions, similar mechanisms? Genes Dev 2004;18:204659.

71. Gill G. Something about SUMO inhibits transcription. Curr Opin Genet Dev 2005;15:536-41.

72. Deng Z, Wan M, Sui G. PIASy-mediated sumoylation of Yin Yang 1 depends on their interaction but not the RING finger. Mol Cell Biol 2007;27:3780-92.

73. Hiromura M, Choi CH, Sabourin NA, Jones H, Bachvarov D, Usheva A. YY1 is regulated by O-linked Nacetylglucosaminylation (O-glcNAcylation). J Biol Chem 2003;278:14046-52.

74. Jackson SP, Tjian R. O-glycosylation of eukaryotic transcription factors: implications for mechanisms of transcriptional regulation. Cell 1988;55:125-33.

75. Griesenbeck J, Ziegler M, Tomilin N, Schweiger M, Oei SL. Stimulation of the catalytic activity of poly(ADPribosyl) transferase by transcription factor Yin Yang 1. FEBS Lett 1999;443:20-4.
76. Oei SL, Shi Y. Poly(ADP-ribosyl)ation of transcription factor Yin Yang 1 under conditions of DNA damage. Biochem Biophys Res Commun 2001;285:27-31.

77. Eliassen KA, Baldwin A, Sikorski EM, Hurt MM. Role for a YY1-binding element in replication-dependent mouse histone gene expression. Mol Cell Biol 1998;18:7106-18.

78. Last TJ, van Wijnen AJ, Birnbaum MJ, Stein GS, Stein JL. Multiple interactions of the transcription factor YY1 with human histone $\mathrm{H} 4$ gene regulatory elements. J Cell Biochem 1999;72:507-16.

79. Inouye CJ, Seto E. Relief of YY1-induced transcriptional repression by protein-protein interaction with the nucleolar phosphoprotein B23. J Biol Chem 1994;269:6506-10.

80. Weng JJ, Yung BY. Nucleophosmin/B23 regulates PCNA promoter through YY1. Biochem Biophys Res Commun 2005;335:826-31.

81. Srinivasan L, Atchison ML. YY1 DNA binding and PcG recruitment requires CtBP. Genes Dev 2004;18:2596601.

82. Atchison L, Ghias A, Wilkinson F, Bonini N, Atchison ML. Transcription factor YY1 functions as a PcG protein in vivo. EMBO J 2003;22:1347-58.

83. Garcia E, Marcos-Gutierrez C, del Mar Lorente M, Moreno JC, Vidal M. RYBP, a new repressor protein that interacts with components of the mammalian Polycomb complex, and with the transcription factor YY1. EMBO J 1999;18:3404-18.

84. Schlisio S, Halperin T, Vidal M, Nevins JR. Interaction of $\mathrm{YY} 1$ with E2Fs, mediated by RYBP, provides a mechanism for specificity of E2F function. EMBO J 2002;21:5775-86.

85. Chen D, Zhang J, Li M, Rayburn ER, Wang H, Zhang R. RYBP stabilizes p53 by modulating MDM2. EMBO Rep 2009;10:166-72.

86. Huang NE, Lin CH, Lin YS, Yu WC. Modulation of YY1 activity by SAP30. Biochem Biophys Res Commun 2003;306:267-75.

87. Le May N, Mansuroglu Z, Leger P, et al. A SAP30 complex inhibits IFN-beta expression in Rift Valley fever virus infected cells. PLoS Pathog 2008;4:e13.

88. Cai Y, Jin J, Yao T, et al. YY1 functions with INO80 to activate transcription. Nat Struct Mol Biol 2007;14:872-4. 89. Wu S, Shi Y, Mulligan P, et al. A YY1-INO80 complex regulates genomic stability through homologous recombination-based repair. Nat Struct Mol Biol 2007;14:1165-72.

90. Gordon SJ, Saleque S, Birshtein BK. Yin Yang 1 is a lipopolysaccharide-inducible activator of the murine 3' Igh enhancer, hs3. J Immunol 2003;170:5549-57.

91. Petkova V, Romanowski MJ, Sulijoadikusumo I, et al. Interaction between YY1 and the retinoblastoma protein. Regulation of cell cycle progression in differentiated cells. J Biol Chem 2001;276:7932-6.

92. Shrivastava A, Yu J, Artandi S, Calame K. YY1 and cMyc associate in vivo in a manner that depends on c-Myc levels. Proc Natl Acad Sci USA 1996;93:10638-41. 
93. Vernon EG, Gaston K. Myc and YY1 mediate activation of the Surf-1 promoter in response to serum growth factors. Biochim Biophys Acta 2000;1492:172-9.

94. Kang JH, Chang SY, Yeom DH, Kim SA, Um SJ, Hong KJ. Weakening of the repressive YY-1 site on the thrombospondin-1 promoter via c-Jun/YY-1 interaction. Exp Mol Med 2004;36:300-10.

95. Krippner-Heidenreich A, Walsemann G, Beyrouthy MJ, et al. Caspase-Dependent Regulation and Subcellular Redistribution of the Transcriptional Modulator YY1 during Apoptosis. Mol Cell Biol 2005;25:3704-14.

96. Usheva A, Shenk T. YY1 transcriptional initiator: protein interactions and association with a DNA site containing unpaired strands. Proc Natl Acad Sci USA 1996;93:13571-6.

97. Seto E, Lewis B, Shenk T. Interaction between transcription factors Sp1 and YY1. Nature 1993;365:462-4. 98. Austen M, Luscher B, Luscher-Firzlaff JM. Characterization of the transcriptional regulator YY1. The bipartite transactivation domain is independent of interaction with the TATA box-binding protein, transcription factor IIB, TAFII55, or cAMP-responsive element-binding protein (CPB)-binding protein. J Biol Chem 1997;272:1709-17.

99. Renna M, Caporaso MG, Bonatti S, Kaufman RJ, Remondelli P. Regulation of ergic-53 gene transcription in response to endoplasmic reticulum stress. J Biol Chem 2007;282:22499-512.

100. Flanagan JR. Autologous stimulation of YY1 transcription factor expression: role of an insulin-like growth factor. Cell Growth Differ 1995;6:185-90.

101. Santiago FS, Lowe HC, Bobryshev YV, Khachigian LM. Induction of the transcriptional repressor Yin Yang-1 by vascular cell injury. Autocrine/paracrine role of endogenous fibroblast growth factor-2. J Biol Chem 2001;276:41143-9.

102. Li H, Liu H, Wang $\mathrm{Z}$, et al. The role of transcription factors $\mathrm{Sp} 1$ and $\mathrm{YY} 1$ in proximal promoter region in initiation of transcription of the mu opioid receptor gene in human lymphocytes. J Cell Biochem 2008;104:237-50.

103. Hara Y, Kusumi Y, Mitsumata M, Li XK, Fujino M. Lysophosphatidylcholine upregulates LOX-1, chemokine receptors, and activation-related transcription factors in human T-cell line Jurkat. J Thromb Thrombolysis 2008;26:113-8.

104. Wang H, Hertlein E, Bakkar N, et al. NF-kappaB regulation of YY1 inhibits skeletal myogenesis through transcriptional silencing of myofibrillar genes. Mol Cell Biol 2007;27:4374-87.

105. Huerta-Yepez S, Vega M, Garban H, Bonavida B. Involvement of the TNF-alpha autocrine-paracrine loop, via NF-kappaB and YY1, in the regulation of tumor cell resistance to Fas-induced apoptosis. Clin Immunol 2006;120:297-309.

106. Vega MI, Jazirehi AR, Huerta-Yepez S, Bonavida B. Rituximab-induced inhibition of YY1 and Bcl-xL expression in Ramos non-Hodgkin's lymphoma cell line via inhibition of NF-kappaB activity: role of YY1 and Bcl-xL in Fas resistance and chemoresistance, respectively. $J$ Immunol 2005;175:2174-83.

107. Joshi B, Rastogi S, Morris M, et al. Differential regulation of human YY1 and caspase 7 promoters by prohibitin through E2F1 and p53 binding sites. Biochem J 2007;401:155-66.

108. Baritaki S, Katsman A, Chatterjee D, Yeung KC, Spandidos DA, Bonavida B. Regulation of tumor cell sensitivity to TRAIL-induced apoptosis by the metastatic suppressor Raf kinase inhibitor protein via Yin Yang 1 inhibition and death receptor 5 up-regulation. J Immunol 2007;179:5441-53.

109. Huerta-Yepez S, Vega M, Escoto-Chavez SE, et al. Nitric oxide sensitizes tumor cells to TRAIL-induced apoptosis via inhibition of the DR5 transcription repressor Yin Yang 1. Nitric Oxide 2009;20:39-52.

110. Hongo F, Garban H, Huerta-Yepez S, et al. Inhibition of the transcription factor Yin Yang 1 activity by Snitrosation. Biochem Biophys Res Commun 2005;336:692701.

111. Porichis F, Morou A, Baritaki S, Spandidos DA, Krambovitis E. Activation-induced cell death signalling in CD4+ T cells by staphylococcal enterotoxin A. Toxicol Lett 2008;176:77-84.

112. Joo $\mathrm{M}$, Wright JG, $\mathrm{Hu} \mathrm{NN}$, et al. Yin Yang 1 enhances cyclooxygenase-2 gene expression in macrophages. Am J Physiol Lung Cell Mol Physiol 2007;292:L1219-26.

113. Xie J, Briggs JA, Briggs RC. Human hematopoietic cell specific nuclear protein MNDA interacts with the multifunctional transcription factor YY1 and stimulates YY1 DNA binding. J Cell Biochem 1998;70:489-506.

114. Seligson D, Horvath S, Huerta-Yepez S, et al. Expression of transcription factor Yin Yang 1 in prostate cancer. Int J Oncol 2005;27:131-41.

115. Erkeland SJ, Valkhof M, Heijmans-Antonissen C, et al. The gene encoding the transcriptional regulator Yin Yang 1 (YY1) is a myeloid transforming gene interfering with neutrophilic differentiation. Blood 2003;101:1111-7.

116. de Nigris F, Botti C, de Chiara A, et al. Expression of transcription factor Yin Yang 1 in human osteosarcomas. Eur J Cancer 2006;42:2420-4.

117. de Nigris F, Botti C, Rossiello R, Crimi E, Sica V, Napoli C. Cooperation between Myc and YY1 provides novel silencing transcriptional targets of alpha3beta1integrin in tumour cells. Oncogene 2007;26:382-94.

118. Baritaki S, Sifakis S, Huerta-Yepez S, et al. Overexpression of VEGF and TGF-beta1 mRNA in Pap smears correlates with progression of cervical intraepithelial neoplasia to cancer: implication of YY1 in cervical tumorigenesis and HPV infection. Int J Oncol 2007;31:69-79.

119. Baritaki S, Chatzinikola AM, Vakis AF, et al. YY1 over-expression in human brain gliomas and meningiomas correlates with TGF-beta1, IGF-1 and FGF-2 mRNA levels. Cancer Invest 2009;27:184-92.

120. Berchuck A, Iversen ES, Lancaster JM, et al. Patterns of gene expression that characterize long-term 
survival in advanced stage serous ovarian cancers. Clin Cancer Res 2005;11:3686-96.

121. Sakhinia E, Glennie C, Hoyland JA, et al. Clinical quantitation of diagnostic and predictive gene expression levels in follicular and diffuse large B-cell lymphoma by RT-PCR gene expression profiling. Blood 2007;109:3922-8. 122. Chinnappan D, Xiao D, Ratnasari A, Andry C, King TC, Weber HC. Transcription factor YY1 expression in human gastrointestinal cancer cells. Int $\mathrm{J}$ Oncol 2009;34:1417-23.

123. de Nigris F, Rossiello R, Schiano C, et al. Deletion of Yin Yang 1 protein in osteosarcoma cells on cell invasion and CXCR4/angiogenesis and metastasis. Cancer Res 2008;68:1797-808.

124. Thomassen M, Tan Q, Kruse TA. Gene expression meta-analysis identifies metastatic pathways and transcription factors in breast cancer. BMC Cancer 2008;8:394.

125. Matsumura N, Huang Z, Baba T, et al. Yin yang 1 modulates taxane response in epithelial ovarian cancer. Mol Cancer Res 2009;7:210-20.

126. Bracken AP, Pasini D, Capra M, Prosperini E, Colli E, Helin K. EZH2 is downstream of the pRB-E2F pathway, essential for proliferation and amplified in cancer. EMBO J 2003;22:5323-35.

127. Simon JA, Lange CA. Roles of the EZH2 histone methyltransferase in cancer epigenetics. Mutat Res 2008;647:21-9.

128. Varambally S, Dhanasekaran SM, Zhou M, et al. The polycomb group protein EZH2 is involved in progression of prostate cancer. Nature 2002;419:624-9.

129. Berezovska OP, Glinskii AB, Yang Z, Li XM, Hoffman RM, Glinsky GV. Essential role for activation of the Polycomb group (PcG) protein chromatin silencing pathway in metastatic prostate cancer. Cell Cycle 2006;5:1886-901.

130. Chen Z, Trotman LC, Shaffer D, et al. Crucial role of p53-dependent cellular senescence in suppression of Ptendeficient tumorigenesis. Nature 2005;436:725-30.

131. Tang X, Milyavsky M, Shats I, Erez N, Goldfinger N, Rotter V. Activated p53 suppresses the histone methyltransferase EZH2 gene. Oncogene 2004;23:5759-69. 132. Yu J, Yu J, Rhodes DR, et al. A polycomb repression signature in metastatic prostate cancer predicts cancer outcome. Cancer Res 2007;67:10657-63.

133. Bryant RJ, Cross NA, Eaton CL, Hamdy FC, Cunliffe VT. EZH2 promotes proliferation and invasiveness of prostate cancer cells. Prostate 2007;67:547-56.

134. Bryant RJ, Winder SJ, Cross SS, Hamdy FC, Cunliffe VT. The polycomb group protein EZH2 regulates actin polymerization in human prostate cancer cells. Prostate 2008;68:255-63.

135. Fearon ER, Vogelstein B. A genetic model for colorectal tumorigenesis. Cell 1990;61:759-67.

136. Arends JW. Molecular interactions in the Vogelstein model of colorectal carcinoma. J Pathol 2000;190:412-6.

137. Bonavida B, Huerta-Yepez S, Goodglick L, Mizutani Y, Miki T. Can we develop biomarkers that predict response of cancer patients to immunotherapy? Biomarkers 2005;10 Suppl 1:69-76.

138. Vega MI, Huerta-Yepez S, Jazirehi AR, Garban H, Bonavida B. Rituximab (chimeric anti-CD20) sensitizes BNHL cell lines to Fas-induced apoptosis. Oncogene 2005;24:8114-27.

139. Hsu KW, Hsieh RH, Lee $\mathrm{YH}$, et al. The activated Notch1 receptor cooperates with alpha-enolase and MBP-1 in modulating c-myc activity. Mol Cell Biol 2008;28:482942.

140. Li WW, Hsiung Y, Zhou Y, Roy B, Lee AS. Induction of the mammalian GRP78/BiP gene by $\mathrm{Ca} 2+$ depletion and formation of aberrant proteins: activation of the conserved stress-inducible grp core promoter element by the human nuclear factor YY1. Mol Cell Biol 1997;17:54-60.

141. Mao C, Tai WC, Bai Y, Poizat C, Lee AS. In vivo regulation of Grp78/BiP transcription in the embryonic heart: role of the endoplasmic reticulum stress response element and GATA-4. J Biol Chem 2006;281:8877-87.

142. Takasaki N, Kurokawa D, Nakayama R, Nakayama J, Aizawa S. Acetylated YY1 regulates Otx2 expression in anterior neuroectoderm at two cis-sites $90 \mathrm{~kb}$ apart. EMBO J 2007;26:1649-59.

143. Di C, Liao S, Adamson DC, et al. Identification of OTX2 as a medulloblastoma oncogene whose product can be targeted by all-trans retinoic acid. Cancer Res 2005;65:919-24.

144. Barrallo-Gimeno A, Nieto MA. The Snail genes as inducers of cell movement and survival: implications in development and cancer. Development 2005;132:3151-61.

145. Satoh K, Ginsburg E, Vonderhaar BK. Msx-1 and Msx-2 in mammary gland development. J Mammary Gland Biol Neoplasia 2004;9:195-205.

146. Hehlgans T, Strominger JL. Activation of transcription by binding of NF-E1 (YY1) to a newly identified element in the first exon of the human DR alpha gene. J Immunol 1995;154:5181-7.

147. Rangel LB, Agarwal R, Sherman-Baust CA, et al. Anomalous expression of the HLA-DR alpha and beta chains in ovarian and other cancers. Cancer Biol Ther 2004;3:1021-7.

148. Brule S, Sayasith K, Sirois J, Silversides DW, Lussier JG. Structure of the bovine VASAP-60/PRKCSH gene, functional analysis of the promoter, and gene expression analysis. Gene 2007;391:63-75.

149. Kim JD, Yu S, Choo JH, Kim J. Two evolutionarily conserved sequence elements for Peg3/Usp29 transcription. BMC Mol Biol 2008;9:108.

150. Abbondanza C, de Nigris F, De Rosa C, Rossiello R, Puca GA, Napoli C. Silencing of YY1 downregulates RIZ1 promoter in human osteosarcoma. Oncol Res 2008;17:3341.

151. Bheda A, Creek KE, Pirisi L. Loss of p53 induces epidermal growth factor receptor promoter activity in normal human keratinocytes. Oncogene 2008;27:4315-23.

152. Athanikar JN, Badge RM, Moran JV. A YY1-binding site is required for accurate human LINE-1 transcription initiation. Nucleic Acids Res 2004;32:3846-55. 
153. Berndt JA, Kim JG, Tosic M, Kim C, Hudson LD. The transcriptional regulator Yin Yang 1 activates the myelin PLP gene. J Neurochem 2001;77:935-42.

154. Buescher JL, Martinez LB, Sato S, Okuyama S, Ikezu T. YY1 and FoxD3 regulate antiretroviral zinc finger protein OTK18 promoter activation induced by HIV-1 infection. J Neuroimmune Pharmacol 2009;4:103-15.

155. Labrie $\mathrm{C}$, Lee $\mathrm{BH}$, Mathews MB. Transcription factors RFX1/EF-C and ATF-1 associate with the adenovirus E1A-responsive element of the human proliferating cell nuclear antigen promoter. Nucleic Acids Res 1995;23:3732-41.

156. Jiang L, Yao M, Shi J, Shen P, Niu G, Fei J. Yin yang 1 directly regulates the transcription of $\mathrm{RE}-1$ silencing transcription factor. J Neurosci Res 2008;86:1209-16.

157. Majumder S. REST in good times and bad: roles in tumor suppressor and oncogenic activities. Cell Cycle 2006;5:1929-35.

158. Jacobsen BM, Skalnik DG. YY1 binds five ciselements and trans-activates the myeloid cell-restricted gp91(phox) promoter. J Biol Chem 1999;274:29984-93.

159. Ushio-Fukai M, Nakamura Y. Reactive oxygen species and angiogenesis: NADPH oxidase as target for cancer therapy. Cancer Lett 2008;266:37-52.

160. Glenn DJ, Wang F, Chen S, Nishimoto M, Gardner DG. Endothelin-stimulated human B-type natriuretic peptide gene expression is mediated by Yin Yang 1 in association with histone deacetylase 2. Hypertension 2009;53:549-55.

161. Holloway K, Sade H, Romero IA, Male D. Action of transcription factors in the control of transferrin receptor expression in human brain endothelium. J Mol Biol 2007;365:1271-84.

162. Natesan S, Gilman MZ. DNA bending and orientation-dependent function of YY1 in the c-fos promoter. Genes Dev 1993;7:2497-509.

163. Klar M, Bode J. Enhanceosome formation over the beta interferon promoter underlies a remote-control mechanism mediated by YY1 and YY2. Mol Cell Biol 2005;25:10159-70.

164. Miao J, Wang Z, Provencher H, et al. HOXB13 promotes ovarian cancer progression. Proc Natl Acad Sci USA 2007;104:17093-8.

165. Liu Q, Merkler KA, Zhang X, McLean MP. Prostaglandin F2alpha suppresses rat steroidogenic acute regulatory protein expression via induction of Yin Yang 1 protein and recruitment of histone deacetylase 1 protein. Endocrinology 2007;148:5209-19.

166. Kim HJ, Kim JE, Ha M, et al. Steroidogenic acute regulatory protein expression in the normal human brain and intracranial tumors. Brain Res 2003;978:245-9.

167. Franchina M, Woo AJ, Dods J, et al. The CD30 gene promoter microsatellite binds transcription factor Yin Yang 1 (YY1) and shows genetic instability in anaplastic large cell lymphoma. J Pathol 2008;214:65-74.

168. Lee BC, Lee TH, Zagozdzon R, Avraham S, Usheva A, Avraham HK. Carboxyl-terminal Src kinase homologous kinase negatively regulates the chemokine receptor CXCR4 through YY1 and impairs CXCR4/CXCL12 (SDF- 1alpha)-mediated breast cancer cell migration. Cancer Res 2005;65:2840-5.

169. He CQ, Ding NZ, Fan W. YY1 repressing peroxisome proliferator-activated receptor delta promoter. Mol Cell Biochem 2008;308:247-52.

170. Crawford EL, Blomquist T, Mullins DN, et al. CEBPG regulates ERCC5/XPG expression in human bronchial epithelial cells and this regulation is modified by E2F1/YY1 interactions. Carcinogenesis 2007;28:2552-9.

171. Tone Y, Kojima Y, Furuuchi K, et al. OX40 Gene Expression Is Up-Regulated by Chromatin Remodeling in Its Promoter Region Containing Sp1/Sp3, YY1, and NF\{kappa\}B Binding Sites. J Immunol 2007;179:1760-7.

172. Redmond WL, Weinberg AD. Targeting OX40 and OX40L for the treatment of autoimmunity and cancer. Crit Rev Immunol 2007;27:415-36.

173. Cicatiello L, Addeo R, Sasso A, et al. Estrogens and progesterone promote persistent CCND1 gene activation during G1 by inducing transcriptional derepression via cJun/c-Fos/estrogen receptor (progesterone receptor) complex assembly to a distal regulatory element and recruitment of cyclin D1 to its own gene promoter. Mol Cell Biol 2004;24:7260-74.

174. Alvarez-Salas LM, Benitez-Hess ML, Dipaolo JA. YY1 and c-Jun transcription factors participate in the repression of the human involucrin promoter. Int $\mathrm{J}$ Oncol 2005;26:259-66.

175. Kobrossy L, Rastegar M, Featherstone M. Interplay between chromatin and trans-acting factors regulating the Hoxd4 promoter during neural differentiation. J Biol Chem 2006.

176. Yang WM, Yao YL, Sun JM, Davie JR, Seto E. Isolation and characterization of cDNAs corresponding to an additional member of the human histone deacetylase gene family. J Biol Chem 1997;272:28001-7.

177. Sucharov CC, Langer S, Bristow M, Leinwand L. Shuttling of HDAC5 in H9C2 cells regulates YY1 function through CaMKIV/PKD and PP2A. Am J Physiol Cell Physiol 2006;291:C1029-37.

178. Kuzmichev A, Jenuwein T, Tempst P, Reinberg D. Different EZH2-containing complexes target methylation of histone $\mathrm{H} 1$ or nucleosomal histone H3. Mol Cell 2004;14:183-93.

179. Filippova GN. Genetics and epigenetics of the multifunctional protein CTCF. Curr Top Dev Biol 2008;80:337-60.

180. Liao WR, Hsieh RH, Hsu KW, et al. The CBF1independent Notch1 signal pathway activates human cmyc expression partially via transcription factor YY1. Carcinogenesis 2007;28:1867-76.

181. Shrivastava A, Saleque S, Kalpana GV, Artandi S, Goff SP, Calame K. Inhibition of transcriptional regulator Yin-Yang-1 by association with c-Myc. Science 1993;262:1889-92.

182. Yeh TS, Lin YM, Hsieh RH, Tseng MJ. Association of transcription factor YY1 with the high molecular weight Notch complex suppresses the transactivation activity of Notch. J Biol Chem 2003;278:41963-9. 
183. Kuzmin A, Han Z, Golding MC, Mann MR, Latham KE, Varmuza S. The PcG gene Sfmbt2 is paternally expressed in extraembryonic tissues. Gene Expr Patterns 2008;8:107-16.

184. Ohtomo T, Horii T, Nomizu M, Suga T, Yamada J. Molecular cloning of a structural homolog of YY1AP, a coactivator of the multifunctional transcription factor YY1. Amino Acids 2007;33:645-52.

185. Wang CY, Liang YJ, Lin YS, Shih HM, Jou YS, Yu WC. YY1AP, a novel co-activator of YY1. J Biol Chem 2004;279:17750-5.

186. Li L, Shi Y, Wu H, et al. Hepatocellular carcinomaassociated gene 2 interacts with MAD2L2. Mol Cell Biochem 2007;304:297-304.

187. Kalenik JL, Chen D, Bradley ME, Chen SJ, Lee TC. Yeast two-hybrid cloning of a novel zinc finger protein that interacts with the multifunctional transcription factor YY1. Nucleic Acids Res 1997;25:843-9.

188. Bannasch D, Madge B, Schwab M. Functional interaction of Yaf2 with the central region of $\mathrm{MycN}$. Oncogene 2001;20:5913-9.

189. Huang WH, Mai RT, Lee YH. Transcription factor YY1 and its associated acetyltransferases CBP and p300 interact with hepatitis delta antigens and modulate hepatitis delta virus RNA replication. J Virol 2008;82:7313-24.

190. Kang HC, Chung BM, Chae JH, Yang SI, Kim CG, Kim CG. Identification and characterization of four novel peptide motifs that recognize distinct regions of the transcription factor CP2. FEBS J 2005;272:1265-77.

191. Lee KH, Evans S, Ruan TY, Lassar AB. SMADmediated modulation of YY1 activity regulates the BMP response and cardiac-specific expression of a GATA4/5/6dependent chick Nkx2.5 enhancer. Development 2004;131:4709-23.

192. Ralph WM, Jr., Liu K, Auborn KJ. CCAAT/enhancerbinding protein beta represses human papillomavirus 11 upstream regulatory region expression through a promoter-proximal YY1-binding site. J Gen Virol 2006;87(Pt 1):51-9. 